\title{
Incidencia de infección nosocomial quirúrgica en ginecología y obstetricia en un hospital comarcal
}

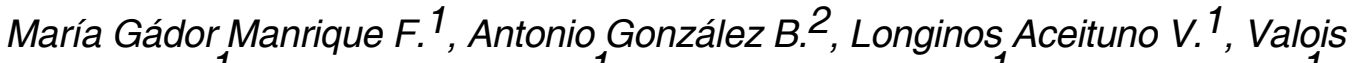 \\ González A. ${ }^{1}$, Rosario Redondo A. ${ }^{1}$, Leonardo Mauro Aisa ${ }^{1}$, Luís Delgado M. ${ }^{1}$ \\ ${ }^{1}$ Servicio de Obstetricia y Ginecología, ${ }^{2}$ Servicio de Medicina Preventiva. Hospital La Inmaculada. Huércal-Overa, \\ Almería-Andalucía. España.
}

\section{RESUMEN}

Introducción: Las infecciones nosocomiales en Obstetricia y Ginecología son causa importante de morbilidad y mortalidad, siendo las más frecuente las de localización quirúrgica. Objetivo: Analizar la incidencia de infecciones nosocomiales relacionadas con las intervenciones mayores más frecuentemente realizadas en Obstetricia y Ginecología. Métodos: Estudio prospectivo de 715 pacientes intervenidas de cesárea o histerectomía abdominal o vaginal, en el Hospital La Inmaculada de Huércal-Overa (Almería, España) en el periodo comprendido entre el 31 de octubre de 2008 y de 30 de agosto de 2011. Se han analizado como variables la Incidencia Acumulada y la Densidad de Incidencia para cada tipo de infección nosocomial detectada, independientemente para cada tipo de intervención. También se han calculado las incidencias ajustadas por el índice NNIS (Nacional Nosocomial Infection Surveillance System). Resultados: Se detectaron 30 infecciones nosocomiales $(4,2 \%)$. La infección nosocomial diagnosticada con mayor frecuencia fue la endometritis tras cesárea, siendo el microorganismo más frecuentemente detectado la Eschericia Coli. Conclusiones: Las infecciones nosocomiales, en concreto las infecciones de localización quirúrgica, son un problema potencialmente grave y relativamente frecuente, que se relaciona con una mayor morbilidad, siendo importante realizar una profilaxis adecuada y una correcta vigilancia para el diagnóstico e instauración precoz del tratamiento.

\section{PALABRAS CLAVE: Infección nosocomial, herida quirúrgica, cesárea, histerectomía}

\section{SUMMARY}

Introduction: Nosocomial infections in obstetrics and gynecology are an important cause of morbidity and mortality, the most frequent in the surgical site. Objective: To analyze the incidence of nosocomial infections related to major interventions most frequently performed in the service of Obstetrics and Gynaecology. Methods: Prospective study of 715 patients undergoing surgery, of caesarean section and abdominal or vaginal hysterectomy at the Hospital La Inmaculada of Huercal-Overa (Almería, Spain) in the period from October 31, 2008 and August 30, 2011. Variables were analyzed as Cumulative Incidence and Incidence Density for each type of nosocomial infection detected, separately for each type of intervention. Also calculated adjusted incidences NNIS index (Nacional Nosocomial Infection Surveillance System). Results: We detected 30 nosocomial infections (4.2\%). The nosocomial infection most frequently diagnosed in our study was the endometritis after cesarean, being the most frequent microorganism detected E. Coli. Conclusions: Nosocomial infections, particularly surgical site infections are a potentially serious problem and relatively frequent, which is related to increased morbidity, is important to perform adequate prophylaxis and make a correct surveillance for diagnosis and an early start of treatment.

KEY WORDS: Nosocomial infection, surgical wound, cesarean, hysterectomy 


\section{INTRODUCCIÓN}

Según la OMS la infección nosocomial (IN) se puede definir como una infección contraída en el hospital por un paciente hospitalizado, la cual no se había manifestado ni estaba en período de incubación en el momento del ingreso. Comprende también las infecciones contraídas en el hospital, pero que debutan después del alta hospitalaria (1).

Pese al importante avance en tratamientos antibióticos y al mayor grado de control, la IN sigue siendo relevante. Se calcula que un promedio de $8,7 \%$ de los pacientes hospitalizados van a desarrollar alguna IN, siendo muy variable en función del hospital, de la especialidad y del tipo de pacientes atendidos. La tasa de mortalidad puede llegar al 1 - $4 \%$ (2).

Entre los pacientes intervenidos, las IN suponen la complicación más frecuente, y la que se relaciona con una mayor morbimortalidad, y por consiguiente una mayor estancia hospitalaria y un mayor gasto sanitario.

En EEUU se estima que la infecciones nosocomiales quirúrgicas (INQ) son responsables de 20.000 muertes hospitalarias anuales, y producen un incremento de la estancia hospitalaria en 7,3 días con un coste adicional de unos 3000 dólares por paciente (3).

Por ello supone una prioridad establecer medidas para su disminución, tales como: vigilancia epidemiológica: el estudio EPINE (4) (Estudio de la Prevalencia de la Infección Nosocomial en España) realiza anualmente una encuesta transversal para determinar la prevalencia de IN en cada centro, programas de prevención, e identificación precoz de las IN instaurando el tratamiento antibiótico adecuado cuanto antes. La implantación de sistemas adecuados de vigilancia y control consigue reducir las cifras globales de incidencia de INQ entre 19 y $41 \%(5,6)$.

La prevalencia de INQ varía en función del tipo de cirugía (limpia, limpia-contaminada, contaminada o sucia). Se considera que las intervenciones quirúrgicas mayores más frecuentemente realizadas en ginecología y obstetricia (cesáreas e histerectomías) son principalmente cirugía limpia-contaminada (7), o contaminada en casos de cirugía vaginal.

En España se calcula una incidencia de INQ en Obstetricia y Ginecología cercana al 5\% (8). Las IN en este ámbito se dividen en:

- Infección de localización quirúrgica (ILQ): secreción purulenta, absceso o celulitis difusa en el sitio de la intervención quirúrgica en el curso de un mes posterior a la cirugía (ILQ superficial, ILQ profunda y ILQ de órgano).

- Infección urinaria: cultivo de orina positivo, al menos con 105 ufc/ml.

- Infección respiratoria: presenta 2 de los siguientes signos-síntomas: tos, esputo purulento, nuevo infiltrado en radiografía de tórax compatible con infección.

- Infección del sitio de inserción de un catéter intravascular, detectado clínicamente.

- Septicemia/bacteriemia: fiebre o escalofríos, y hemocultivo positivo.

- Endometritis: cultivo de loquios positivo o confirmación anatomopatológica.

En Obstetricia y Ginecología, las INQ corresponden, en la mayoría de los casos, a patología polimicrobiana, siendo las más frecuentes la endometritis y la infección de la herida quirúrgica superficial.

El objetivo del estudio fue analizar la incidencia de IN relacionadas con las intervenciones más frecuentemente realizadas en el servicio de Obstetricia y Ginecología: cesáreas, histerectomías abdominales (incluyendo radicales) e histerectomías vaginales.

\section{PACIENTES Y MÉTODOS}

Estudio de cohortes prospectivo sobre las pacientes intervenidas de cesáreas o histerectomías abdominales (incluyendo radicales) o vaginales, durante el periodo del 31 de Octubre de 2008 hasta el 30 de Agosto de 2011, en el Servicio de Obstetricia y Ginecología del Hospital La Inmaculada de Huércal-Overa, Almería-Andalucía (España).

Se han analizado las siguientes variables: tipo de ingreso (urgente o programado), riesgo de base (índice ASA) (9), grado de contaminación de la cirugía, técnica quirúrgica, duración de la intervención, profilaxis antibiótica perioperatoria, microorganismos aislados en cultivos.

La recogida de información se ha realizado por el epidemiólogo del Servicio de Medicina Preventiva del Hospital, obteniendo datos de la historia clínica, de la observación del propio paciente o a través de consultas con el personal facultativo del Servicio de Obstetricia y Ginecología.

Se realizó profilaxis antibiótica en todas las intervenciones analizadas, con 2 gramos de Amoxicilina-Clavulánico en las cesáreas y con 2 gramos de Cefazolina en las histerectomias, administrados en los 30 minutos anteriores a la cirugía, en dosis única (10). El lavado quirúrgico de piel se realiza con clorhexidina alcohólica al $2 \%$ y el de vagina con clorhexidina acuosa. Se administra heparina de bajo peso molecular, como profilaxis tromboembólica, durante 7 días. El sondaje vesical es retirado a las 6-12 horas tras la cesárea y a las 24 horas tras la histerectomía. El apósito que cubre la herida es retirado a las 48 horas en ambos casos, la herida no recibe ningún cuidado especial posteriormente.

Tras la intervención, la paciente recibió tratamiento antibiótico empírico de amplio espectro en casos de fiebre $>38^{\circ} \mathrm{C}$ (exceptuando las primeras 
24 horas), tras investigación del posible foco, realización de hemocultivo y urocultivo, siendo los antibióticos más frecuentemente utilizados la Gentamicina y la Clindamicina.

Se calcularon las siguientes medidas: la incidencia acumulada (IA) de IN (IA= enfermas intervenidas que desarrollan IN / total de enfermas intervenidas $x$ 100) y la densidad de incidencia (DI) de IN (DI= infecciones nosocomiales que han desarrollado las pacientes intervenidas / días de estancia totales de las pacientes $\times 1000)$.

Dichas medidas fueron calculadas para la infección de localización quirúrgica (superficial, profunda y de órgano), infección urinaria y bacteriemia, independientemente para cada tipo de intervención (cesárea, histerectomía abdominal e histerectomía vaginal).

También se realizó el cálculo de las cifras de incidencia ajustadas por el índice NNIS, que define el riesgo de tener infección quirúrgica basándose en la determinación de tres parámetros: grado de contaminación de la cirugía, riesgo ASA (American Society Anesthesiologists) y duración de la intervención (9).

\section{RESULTADOS}

Se realizaron un total de 1942 intervenciones en el periodo estudiado, incluyendo cirugía ginecológica, oncológica, vaginal, endoscópica, mamaria y cesáreas. Entre ellas, las cesáreas representan el $26,67 \%$ de las intervenciones, y las histerectomías abdominales y vaginales, el $7,98 \%$ y $2,06 \%$, respectivamente.

En ese periodo se recogieron datos de 715 pacientes intervenidas de cesáreas o histerectomías (Tabla I), detectándose 30 IN, con una incidencia del $4,2 \%$.

El tipo de ingreso fue programado en el $98,1 \%$ de las histerectomías y urgente en el $73,35 \%$ de las cesáreas. El riesgo de base fue leve en la mayoría de los casos $(97,48 \%)$, y moderado en el $2,37 \%$ de las pacientes intervenidas, sólo se intervino una paciente con riesgo de base grave.

En relación al grado de contaminación, se realizaron intervenciones limpias-contaminadas en la mayoría de los casos, lo que corresponde a cesáreas e histerectomías abdominales. Las histerectomías vaginales corresponden a cirugía contaminada, y se detectó un caso de cirugía sucia por perforación de mucosa rectal durante una histerectomía vaginal (Tabla II).

En las 520 cesáreas, se detectaron 16 IN, de las cuales 9 fueron ILQ: 5 superficiales, 1 profunda y 3 de órgano. Se diagnosticaron además 7 endometritis.

En las 155 histerectomías abdominales, se detectaron un total de 13 IN, de las cuales 10 fueron
Tabla I

\section{INTERVENCIONES REALIZADAS OBJETO DE ESTUDIO}

\begin{tabular}{lc}
\hline Intervenciones & $\mathrm{n}$ \\
\hline Cesáreas & 520 \\
Histerectomías abdominales & 155 \\
Histerectomías vaginales & 40 \\
Total & 715 \\
\hline
\end{tabular}

\section{Tabla II \\ INTERVENCIONES SEGÚN GRADO DE CONTAMINACIÓN DE LA CIRUGÍA}

\begin{tabular}{lc}
\hline Tipo de intervención & $\mathrm{n}$ \\
\hline $\begin{array}{l}\text { Limpia-contaminada (cesáreas e } \\
\text { histerectomías abdominales) }\end{array}$ & 670 \\
$\begin{array}{l}\text { Contaminada (histerectomías } \\
\text { vaginales) }\end{array}$ & 44 \\
Sucia & 1 \\
\hline
\end{tabular}

ILQ: 3 superficiales, 3 profundas y 4 de órgano, se diagnosticaron además 2 infecciones de orina y 1 bacteriemia secundaria. Se realizaron 40 histerectomías vaginales, detectándose 1 IN, dicha infección correspondió a una infección urinaria (Tabla III).

La incidencia acumulada (IA) global de IN, en el periodo analizado, fue $4,2 \%$ de las pacientes intervenidas (Tabla IV), y la densidad de incidencia (DI) 8,52 episodios por 1000 pacientes-día.

En cesáreas, la IA de IN fue del 3,07\% (Tabla IV), la IA de ILQ fue $1,73 \%$, la IA de infección de tracto urinario y de bacteriemia fue $0 \%$. La incidencia de IN en cesáreas según las categorías del índice de riesgo de National Nosocomial Infections Surveillance (NNIS) se presenta en la Tabla V.

En relación a las histerectomías abdominales la IA de IN fue $8,38 \%$ (Tabla IV). La IA de ILQ fue $6,45 \%$. La IA de infección de tracto urinario fue $1,29 \%$ y de bacteriemia $0,64 \%$. La incidencia de IN en histerectomías abdominales según las categorías del índice de riesgo de NNIS, y la evolución en los últimos años, se presenta en la Tabla VI.

En cuanto a las histerectomías vaginales la IA de IN fue $2,5 \%$ (Tabla IV), que corresponde con la IA de infección de tracto urinario. La IA de ILQ fue $0 \%$. En el $92,52 \%$ de las intervenciones se realizó profilaxis antibiótica quirúrgica, siendo ésta adecuada en el $96,35 \%$ de las ocasiones.

De los antibióticos empleados de forma empírica, el más utilizado fue la Clindamicina en el periodo $2008-2009(34,78 \%)$ y la Gentamicina en el periodo 2010-2011 (31,03\%). 
Tabla III

\section{LOCALIZACIÓN DE INFECCIÓN NOSOCOMIAL POR TIPO DE INTERVENCIÓN QUIRÚRGICA}

\begin{tabular}{lcccc}
\hline Localización & Cesáreas & $\begin{array}{c}\text { Histerectomías } \\
\text { abdominales }\end{array}$ & $\begin{array}{c}\text { Histerectomías } \\
\text { vaginales }\end{array}$ & Total \\
\hline ILQ superficial & 5 & 3 & 0 & 8 \\
ILQ profunda & 1 & 3 & 0 & 4 \\
ILQ órgano & 3 & 4 & 0 & 7 \\
Endometritis & 7 & 0 & 0 & 7 \\
Infección urinaria & 0 & 2 & 1 & 3 \\
Bacteriemia secundaria & 0 & 1 & 0 & 1 \\
Total & 16 & 13 & 1 & 30 \\
\hline
\end{tabular}

ILQ: Infección de localización.

Tabla IV

INCIDENCIA ACUMULADA DE INFECCIÓN NOSOCOMIAL: 2008-2011

\begin{tabular}{lr}
\hline Incidencia acumulada & $2008-2011$ \\
\hline Global de infección nosocomial & $4,20 \%(30 / 715)$ \\
Infección nosocomial en cesáreas & $3,07 \%(16 / 520)$ \\
Infección nosocomial en histerectomía & $8,38 \%(13 / 155)$ \\
abdominales & \\
Infección nosocomial en histerectomía & $2,5 \%(1 / 40)$ \\
vaginales & \\
\hline
\end{tabular}

Tabla V

INCIDENCIA DE INFECCIÓN NOSOCOMIAL QUIRÚRGICA EN CESÁREAS SEGÚN LAS CATEGORÍAS DEL ÍNDICE DE RIESGO NNIS

\begin{tabular}{lc}
\hline Infección nosocomial quirúrgica & Incidencia 2008-2011 \\
\hline Global & 3,07 \\
Índice NNIS 0 & 2,40 \\
Índice NNIS 1 & 3,45 \\
Índice NNIS 2 & -
\end{tabular}

NNIS: National Nosocomial Infections Surveillance.

Se realizaron cultivos en el $79,8 \%$ de las infecciones detectadas. El $83,75 \%$ de los cultivos realizados fueron positivos. Los gérmenes más frecuentemente identificados fueron: E. Coli (n: 6), Enterococcus Faecalis ( $\mathrm{n}: 5)$, Klebsiella Pneumoniae (n: 4), Staphylococcus Aureus (n: 2), Proteus Mirabilis ( $n: 2)$, Streptococcus Anginosus (n: 2), otros: Morganella Morgagni, Pseudomona Aureginosa.
Tabla VI

INCIDENCIA DE INFECCIÓN NOSOCOMIAL QUIRÚRGICA EN HISTERECTOMÍAS ABDOMINALES SEGÚN LAS CATEGORÍAS DEL ÍNDICE DE RIESGO NNIS

\begin{tabular}{lc}
\hline Infección nosocomial quirúrgica & Incidencia 2008-2011 \\
\hline Global & 8,38 \\
Índice NNIS 0 & - \\
Índice NNIS 1 & 7,04 \\
Índice NNIS 2 & 14,5 \\
\hline
\end{tabular}

NNIS: National Nosocomial Infections Surveillance.

\section{DISCUSIÓN}

Del 31 de Octubre de 2008 al 30 de Agosto de 2011, en el Servicio de Obstetricia y Ginecología del Hospital La Inmaculada de Huércal-Overa, se han estudiado un total de 715 pacientes intervenidas de cesáreas e histerectomías. Se han detectado un total de 30 infecciones nosocomiales, lo que supone una incidencia del $4,20 \%$, tasas similares a los datos recogidos actualmente en servicios de Obstetricia y Ginecología de hospitales españoles (4).

El riesgo de base se ha considerado leve en el $97,48 \%$ de las intervenciones realizadas. En cuanto al tipo de ingreso, ha sido programado en el $98,1 \%$ de las histerectomías y urgente en el $73,35 \%$ de las cesáreas.

Las infecciones nosocomiales más incidentes han sido las endometritis tras cesáreas, seguidas de infecciones de localización quirúrgica superficial. Es de destacar la baja incidencia de bacteriemias (un caso en el periodo referido) y de infecciones urinarias (también un sólo caso), lo que podría estar en relación con la retirada precoz de la sonda vesical. 
La tasa de infección de herida quirúrgica se mantiene dentro de los estándares nacionales en las intervenciones de cesárea (estándar NNIS global España 2006: 2,48). Los indicadores referidos a las intervenciones por histerectomía abdominal se mantienen aún altos en relación a los indicadores estándares (estándar NNIS global España 2006: 2,99) (8).

Es de destacar también, el porcentaje de cultivos realizados en las infecciones detectadas $(79,8 \%)$, y el gran rendimiento obtenido por éstos (83,75\% de cultivos positivos).

\section{CONCLUSIONES}

Las infecciones nosocomiales, especialmente las de localización quirúrgica, son un problema potencialmente grave y relativamente frecuente, que se relaciona con una mayor morbilidad. Por ello es importante realizar una correcta vigilancia para el diagnóstico e instauración precoz del tratamiento, así como una profilaxis adecuada.

\section{REFERENCIAS}

1. Pujol M, Limón E. Epidemiología general de las infecciones nosocomiales. Sistemas y programas de vigilancia. Enferm Infecc Microbiol Clin 2013;31:108-13.

2. Geubbels EL, Nagelkerke NJ, Mintjes-De-Groot AJ, et al. Reduced risk of surgical site infections through surveillance in a network. Int $\mathrm{J}$ Qual Health Care 2006;18:127-33.

3. National Nosocomial Infections Surveillance (NNIS) System Report, data summary from January 1992 through June 2004. Am J Infect Control 2004;32:47085.
4. Informe EPINE (Estudio de Prevalencia de las Infecciones Nosocomiales en España). Sociedad Española de Medicina Preventiva Salud Pública e Higiene. Disponible en: http://www.sempsph.com. 22ํㅡㄹ Estudio. España 2011

5. Llanos Méndez A, Díaz Molina C, Fernández-Crehuet Navajas R. Infección quirúrgica en un hospital de tercer nivel. Estudio de vigilancia prospectivo (20012004). Cir Esp 2010;88:319-27.

6. Dirección General de Asistencia Sanitaria, Subdirección de Programas y Desarrollo, Subdirección de servicio de Protocolos Asistenciales. Plan de vigilancia y control de las infecciones nosocomiales en los hospitales del Servicio Andaluz de Salud. Ed: Servicio Andaluz de Salud. Consejería de Salud: 2002.

7. Olsen MA, Butler AM, Willers DM, Devkota P, Gross GA, Fraser VJ. Risk Factors for surgical site infection after low transverse cesarean section. Infect Control Hosp Epidemiol 2008;29:477-84

8. Bajo JA, Bajo JM, Lailla JM. Vigilancia de Infección nosocomial, indicador de calidad. Capítulo 12. Fundamentos de Ginecología SEGO 2009;12:151-71. Disponible en: http://www2.univadis.net/microsites/ area_salud_mujer/pdfs/12-Vigilancia_de_la_Infeccion_Nosocomial_indicador_de_calidad.pdf

9. Hospital Infections Program. National Center for Infectious diseases, Centers for Disease control and Prevention, Public Health Service, US department of Health and Human Services, Atlanta, Georgia. Semiannual report. Aggregated data from the National Nosocomial Infections Surveillance (NNIS) System. 1999. Disponible en: www.cdc.gov

10. Young BC, Hacker MR, Dodge LE, Golen TH. Timing of antibiotic administration and infectious morbidity following cesarean delivery: incorporating policy change into workflow. Arch Gynecol Obstet. 2012. 285:121924. 